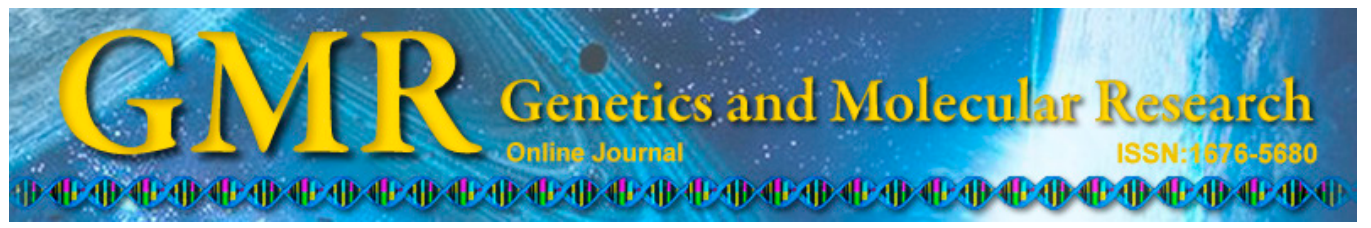

\title{
Clinical applications of internal heat source analysis for breast cancer identification
}

\author{
F. Han, C.W. Liang, G.L. Shi, L. Wang and K.Y. Li \\ Education, Department of Electronic Science and Technology, \\ Corresponding author: K.Y. Li \\ E-mail: 1ky@whu.edu.cn \\ Genet. Mol. Res. 14 (1): 1450-1460 (2015) \\ Received July 17, 2014 \\ Accepted October 23, 2014 \\ Published February 13, 2015 \\ DOI http://dx.doi.org/10.4238/2015.February.13.24
}

Key Laboratory of Artificial Micro and Nano-Structures of the Ministry of School of Physics and Technology, Wuhan University, Wuhan, China

\begin{abstract}
Nondestructive preoperative breast imaging techniques are widely used for breast cancer testing and diagnosis. This study aimed to evaluate the feasibility and efficacy of quantitative diagnosis via the thermal analysis of abnormal metabolism. Nine hundred forty-eight women who underwent breast biopsy from 2009 to 2013 were investigated. Thermal analysis was used to calculate the internal heat source (i.e., tumor) thermal power for each participant. The applicability and effectiveness of our approach were estimated using the chi-square test, kappa statistics $(k)$, and odds ratios (OR). Breast density and tumor size were considered during this estimation. A thermal power $q=0.2 w$ was determined as the optimal separation threshold between breast cancer and benign disease. Moreover, good agreement $(k=0.837)$ with the gold-standard assessment (breast biopsy) was confirmed in $93.2 \%$ of the patients ( $\mathrm{N}=884 / 948)$, and the sensitivity and specificity were 94.2 and $92.9 \%$, respectively. The results also found no significant differences in methodological accuracy between the fatty and dense breasts $(\mathrm{OR}=1.194, \mathrm{P}=0.524)$. Furthermore, after dividing the cohort into three groups according to tumor size $(\mathrm{T} 1:<2 \mathrm{~cm}$; T2: 2 to $5 \mathrm{~cm}$; T3: $>5 \mathrm{~cm}$ ), the tumor size had no effect on the proposed method (ORs $=1, P=0.724)$. Internal heat source analysis can feasibly
\end{abstract}


and efficiently distinguish between breast cancer and benign disease.

Key words: Breast cancer; Diagnosis; Functional imaging;

Heat source analysis; Gold-standard assessment

\section{INTRODUCTION}

Breast cancer is a dreadful and prevalent disease that affects women; this disease has become the second-most important public health problem after skin cancer, affecting 1 in 4 women in the United States (Bezić et al., 2013; Siegel et al., 2013). Surgeons prefer to use advanced imaging modalities when conducting preoperative assessments; however, biopsy remains a gold standard.

To date, various breast imaging technologies have been developed to allow adjuvant diagnoses prior to surgical biopsy. However, the most popular methods, including X-ray mammography, ultrasonic imaging, and breast magnetic resonance imaging, essentially provide morphological and structural information (Gautherie, 1980; Anderson et al., 2005; Aupperlee et al., 2005; AbdullGaffar et al., 2012). However, many types of aggressive tumors containing fewer than 500,000 cells $(<2 \mathrm{~mm}$ in diameter) are likely to pass undetected through most of these scans (Gescheit et al., 2010; Absalan et al., 2012). To exacerbate this problem, a tumor of this size can effectively undergo 19 cell doublings to generate a predicted lethal load of $10^{10}$ to $10^{12}$ cells, which will lead to continued and uncontrollable growth if left untreated (Alroy and Yarden, 2000; Aksel and Mikhailov, 2005; Jemal et al., 2005; Allan et al., 2006). In addition, the use of mammography for clinical diagnosis is restricted under some conditions such as micro-calcifications and extremely dense breasts (Razzaghi et al., 2012; Kim et al., 2013).

The thermal method, by contrast, provides functional information that cannot be easily measured using other methods. This method detects changes in heat generation consequent to abnormal metabolism, which occur 3 to 5 years prior to morphological and structural changes. Skin temperatures are detected using infrared scanners, which offer the advantage of providing thermo-physiological information and indicate early-stage disease in real time (Brown et al., 2006; Chandrasekar et al., 2010; Chavez et al., 2010). These subtle physiological changes might be caused by conditions commonly associated with regional vasodilatation, hyper-perfusion, and hyper-metabolism (Gautherie, 1980; Anbar, 1998; Jones and Plassman, 2002). In addition, during the past few decades, thermal analysis has been investigated as a potential costeffective, noninvasive, and nondestructive diagnostic solution for detecting malignant tumors.

Therefore, a quantitative investigation of thermal pattern information is essential to early breast cancer detection. The first evaluation of thermal conditions in breast cancer was conducted by Lawson and Gaston in 1964. These researchers found that all mammary carcinomas and corresponding venous drainage had elevated temperatures when compared with the corresponding arterial blood supplies (Lawson and Gaston, 1964). In the decades since that investigation, many researchers have devoted their attention to this field. However, most previous studies evaluated qualitative infrared imaging detection methods, although it is difficult to distinguish clearly between benign and malignant tumors using thermal images alone (Liu and Lin, 2010; Ye and Shi, 2013). Today, a somewhat better understanding of thermal pattern changes has been established (Lawson and Alt, 1965; Draper and Jones, 1969; Feasey et al., 1970; Gautherie, 1980; Jones and Plassman, 2002; Chunfang et al., 2005; Gescheit et al., 2010; Zastrow et al., 2010; Absalan et al., 2012; He et al., 2012; Qian et al., 2012; SalmanOgli and Rostami, 2012). Because tumor tissue is generally assumed to exhibit increased 
vascularity and a higher metabolic rate relative to normal tissue, the former is considered to be an internal heat source. Heat conducted from the tumor produces an increase in the prominent surface thermal pattern as generated by the larger vessels (Carter et al., 2000; Choudhury and Kiessling, 2004). The heat produced by the internal source conducts through tissues to reach the body surface, where it produces a hot spot on the dermal surface. Accordingly, the temperature distribution correlates with the source power and depth (Farese and Aebi, 2008; Erb et al., 2010). Consequently, a quantitative assessment of the internal heat source is essential to a clinical breast cancer diagnosis.

In this study, amidst concerns about the early detection and effective treatment of breast cancer, the clinical application of a quantitative diagnosis via thermal analysis was investigated. An internal heat source analysis-based method to determine the optimal threshold for distinguishing breast cancer from benign disease has been proposed in this paper. Using internal heat source analysis, the 2-dimensional (2D) thermal image detected by an infrared camera could be converted to calculate the thermal power of the tumor, which correlates with the malignant or benign nature. The efficiency of this approach was statistically evaluated with respect to breast density and tumor size in a sample of patients from 2009 to 2013.

\section{MATERIAL AND METHODS}

\section{Patient sample}

This prospective study was approved by the Chinese Food and Drug Administration (YZB 0552-2012), and informed consent was obtained from each participant. The study was conducted in compliance with the current local laws of China. Patients (women) who underwent breast biopsy at the People's Hospital of Hubei Province during the period from 2009 to 2013 were investigated to determine whether they met the eligibility criteria for the sample population. Participants were excluded if 1) the diagnosis was not pathologically confirmed via breast biopsy; 2) they had undergone a breast lumpectomy before thermal analysis; 3) their breasts harbored randomly scattered high-temperature surface spots; or 4) the required demographic characteristic data were missing or incomplete. Subsequently, 948 patient specimens were retrospectively evaluated in this study.

\section{Methods}

In this study, a bio-heat model was developed by solving an inverse problem. Given the conduction of heat between the skin temperature and the embedded heat source, a tumor with a higher temperature acts as a heat source within the tissue and causes a hot spot on the dermal surface. An inverse source coefficient problem was established and described according to the well-known Penne's bio-heat transfer equation (Pennes, 1948). Here, the model used for analysis can be described as follows:

$$
\rho c \frac{\partial T}{\partial \tau}=\nabla \cdot k \nabla T+Q_{m}+Q_{b}
$$

(Equation 1)

where $T$ represents the tissue temperature, $\tau$ is time, and $\rho, c$, and $k$ indicate the density, specific heat, and thermal conductivity of the tissue, respectively. The heat source term contains 
2 parts: the metabolic (i.e., $Q_{m}$ ) and perfusion rates (i.e., $Q_{b}$ ).

Because the heat medium in this study was breast tissue, this problem can be simplified based on the following assumptions: i) the tissue is assumed to be homogeneous as well as isotropic with constant properties (i.e., $\rho, c$, and $k$ are constant); and ii) the 2 parts of the heat source term are merged into an equivalent point heat source [i.e., $q \bullet \delta(r)$, where $q$ represents the thermal power]. Subsequently, a semi-analytic method-based steady-state solution to Equation 1 was developed as follows:

$$
T=T_{0}+q / 4 k \pi \sqrt{r^{2}+d^{2}}
$$

where $k, q$, and $d$ represent the thermal conductivity of the breast tissue and the power and depth of the heat source, respectively; $r$ is the radius of the region of interest on the breast surface; and $T$ and $T_{0}$ represent the surface temperature and the undisturbed skin temperature distant from the source, respectively.

According to the above-mentioned model, the thermal power ( $q$ value) of the internal heat source could be fitted with the Levenberg-Marquardt algorithm, based on the breast surface temperature pattern data (Figure 1).

\section{Procedures}

Quantitative breast cancer diagnoses were made by analyzing the internal heat sources embedded in the tissues. Through this analysis, the thermal power ( $q$ value) of the inner source was determined to describe the characteristics of the internal heat source. Contrast trials were previously conducted on large numbers of patients with malignant and benign tumors with the intent to characterize the internal heat sources in both benign and malignant tissue. The applicability and effectiveness of the approach proposed in this paper were estimated via statistical treatments.

All calculations in the analysis were performed based on the surface temperature data, which was obtained via the Thermal Tomography Imaging System (resolution, 320 x 240; noiseequivalent temperature difference, $\leq 50 \mathrm{mK}$ at $25^{\circ} \mathrm{C}$; Patent of China No. ZL200710052685.4 and ZL200720085783.3). Thermometry was conducted in a special room that was designed to maintain a stable environmental temperature $\left(25^{\circ} \mathrm{C}\right)$ and relative humidity (40 to $\left.50 \%\right)$.

\section{Statistical analysis}

The patients' characteristics were analyzed and summarized. The optimal $q$ value threshold for distinguishing breast cancer and benign disease was evaluated using diagnostic evaluation metrics such as accuracy, false-positivity, sensitivity, and specificity. The feasibility and efficacy of the approach proposed in this paper were estimated. The agreement between breast biopsy and the proposed approach was estimated via kappa statistics $(k)$ according to the following definitions of $k$ value strength: $<0.4$, poor agreement; 0.4 to 0.75 , fair agreement; and $\geq 0.75$, excellent agreement. The impacts of the breast density and tumor size on the results were estimated using odds ratios (OR) and 95\% confidence intervals (CI). There were no significant differences between the outcomes of all groups when the OR was near 1 or the 95\% CI included 1. Statistical analyses were performed with SPSS, version 20.0 (SPSS, Inc., 
Chicago, IL, USA), and a 2-tailed P value of $<0.05$ was used to define statistical significance.

\section{RESULTS}

According to the eligibility criteria, 948 women underwent valid analyses during the period from 2009 to 2013. The subjects' demographic characteristics, including the data source, year of diagnosis, age, body mass index, brassiere cup size, family history of breast cancer, menopausal status, and tumor size, are summarized in Table 1.

Most women were between 40 and 69 years of age, had no family history of breast cancer, and had tumors $\leq 5 \mathrm{~cm}$ in size. Overall, among the 948 women, $260(27.4 \%)$ had breast cancer and $688(72.6 \%)$ had benign breast diseases. The results of internal heat source analysis were described as $q$ values (i.e., thermal power). Figure 1 shows the proportion of cases with changes in thermal power. All of the $q$ values fell within an interval of 0 to 0.5 . To investigate the statistical characteristics of $q$, the entire value range was divided into 5 subintervals with interval lengths of 0.1 . The proportion of benign cases decreased from $65 \%$ $(\mathrm{N}=688)$ in the interval of 0 to 0.1 to $2 \%(\mathrm{~N}=688)$ in the interval of 0.3 to 0.4 (Figure 2a). Meanwhile, the proportion of cancer cases increased from $5 \%(\mathrm{~N}=260)$ in the interval of 0.1 to 0.2 to $48 \%(\mathrm{~N}=260)$ in the interval of 0.3 to 0.4 but decreased to $29 \%(\mathrm{~N}=260)$ in the interval of 0.4 to 0.5 .

\section{Table 1. Demographic characteristics of all participants.}

\begin{tabular}{|c|c|}
\hline & No. of patients $(\%)$ \\
\hline Number of participants & 948 \\
\hline \multicolumn{2}{|l|}{ Year } \\
\hline 2009 & $33(3.5 \%)$ \\
\hline 2010 & $241(25.4 \%)$ \\
\hline 2011 & $313(33.0 \%)$ \\
\hline 2012 & $175(18.5 \%)$ \\
\hline 2013 & $186(19.6 \%)$ \\
\hline \multicolumn{2}{|l|}{ Age (years) } \\
\hline$<20$ & $2(0.2 \%)$ \\
\hline $20-29$ & $40(4.2 \%)$ \\
\hline $30-39$ & $168(17.7 \%)$ \\
\hline $40-49$ & $281(29.5 \%)$ \\
\hline $50-59$ & $302(31.9 \%)$ \\
\hline $60-69$ & $136(14.3 \%)$ \\
\hline $70-79$ & $18(1.9 \%)$ \\
\hline$\geq 80$ & $1(0.1 \%)$ \\
\hline \multicolumn{2}{|l|}{ Body mass index $\left(\mathrm{kg} / \mathrm{m}^{2}\right)$} \\
\hline$<25$ & $497(52.4 \%)$ \\
\hline$\geq 25$ & $451(47.6 \%)$ \\
\hline \multicolumn{2}{|l|}{ Brassiere cup size $(\mathrm{cm})$} \\
\hline $\mathrm{A}(2.5)$ & $376(39.7 \%)$ \\
\hline B (5.0) & $483(50.9 \%)$ \\
\hline $\mathrm{C}(7.5)$ & $89(9.4 \%)$ \\
\hline \multicolumn{2}{|c|}{ Family history of breast cancer } \\
\hline Yes & $46(4.9 \%)$ \\
\hline No & $902(95.1 \%)$ \\
\hline \multicolumn{2}{|l|}{ Menopausal status } \\
\hline Premenopausal & $346(36.5 \%)$ \\
\hline Postmenopausal & $602(63.5 \%)$ \\
\hline \multicolumn{2}{|l|}{ Size of tumor } \\
\hline $\mathrm{T} 1(<2 \mathrm{~cm})$ & $94(9.9 \%)$ \\
\hline $\mathrm{T} 2(2-5 \mathrm{~cm})$ & $733(77.3 \%)$ \\
\hline $\mathrm{T} 3(>5 \mathrm{~cm})$ & $121(12.8 \%)$ \\
\hline
\end{tabular}




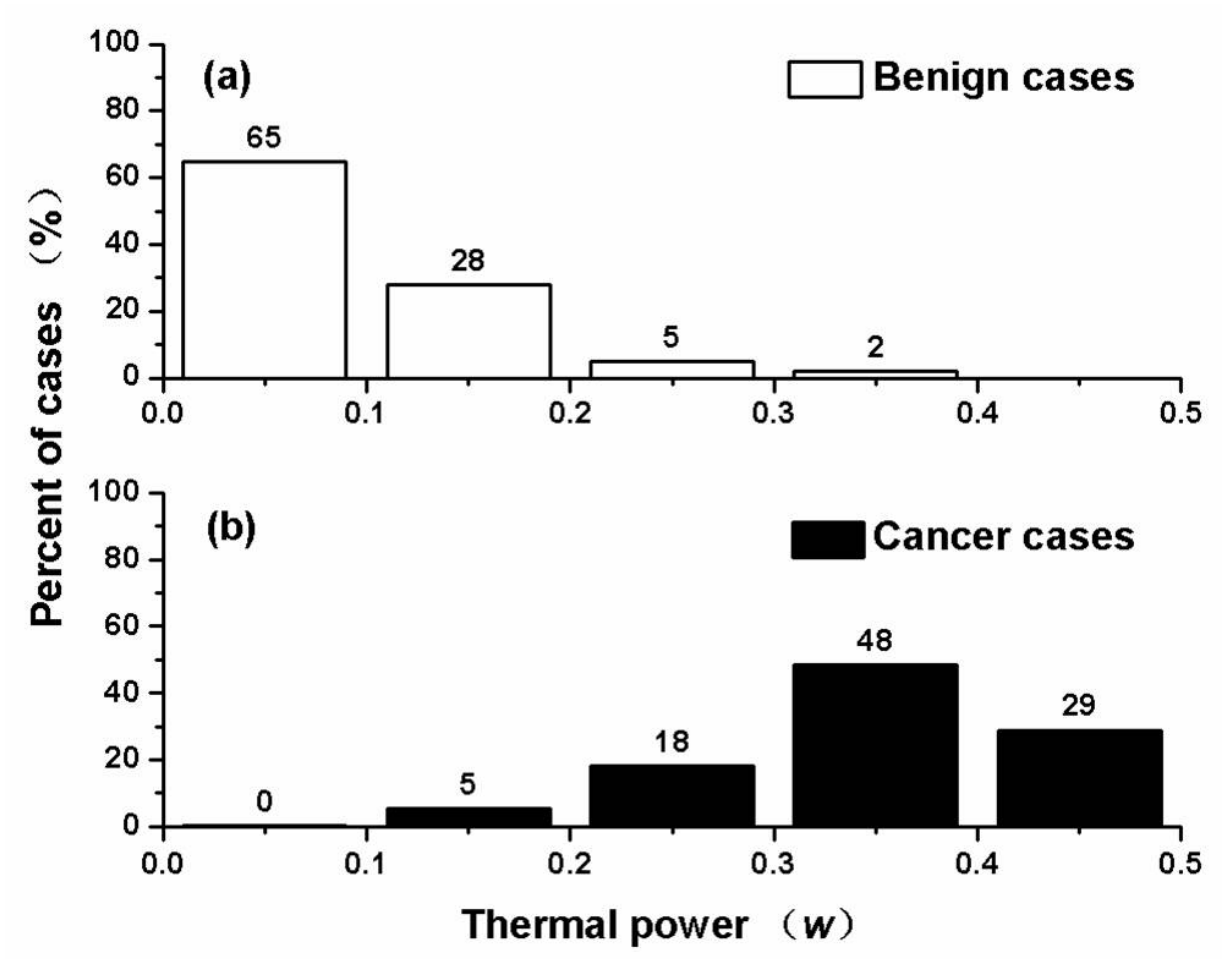

Figure 1. Proportion of cases in each heat source interval according to thermal power ( 0.1 to $0.5 w)$.

According to the above $q$-value distribution, 3 tests with $q$-values of $0.1,0.2$ and 0.3 were performed to identify the best power value fit for distinguishing breast cancer (Figure $2 \mathrm{~b}$, Table 2). The associated error rates of these tests are shown in Table 2. The $q$-value range for benign cases was described as the benign interval, and that for malignant cases was the cancer interval. Note that test 1 exhibited good performance in terms of sensitivity $(99.6 \%)$ but was not extraordinary with regard to specificity (65.1\%) and accuracy (74.6\%). In particular, the false-positive rate $(34.9 \%)$ was extremely poor. Test 3 improved with regard to the false-positive rate $(2.5 \%)$ but decreased in terms of sensitivity (76.2\%). Test 2 exhibited good performance for most metrics (sensitivity, 94.2\%; specificity, 92.9\%; accuracy, 93.2\%) as well as an acceptable false-positive rate (7.1\%). Compared with tests 1 and 3, the approach of test 2 yielded a better overall consideration. Hence, the optimal threshold of $q=0.2 w$ could reasonably distinguish breast cancer from benign disease.

The agreements between the internal heat source analysis and breast biopsy results are cross-tabulated in Table 3. Each patient underwent breast biopsy, and the final outcome was described as "cancer" or "benign". A consistency check involving $k$ was performed. There was substantial agreement between the internal heat source analysis and breast biopsy results $(k=$ $0.837,95 \% \mathrm{CI}=0.781-0.873)$. Overall, $93.2 \%(884 / 948)$ of the assessments exhibited exact agreement. Additionally, the $k$ result was statistically significant $(\mathrm{P}<0.001)$, indicating that the approach in this study showed a good agreement with the gold-standard assessment $(k>0.75)$. 

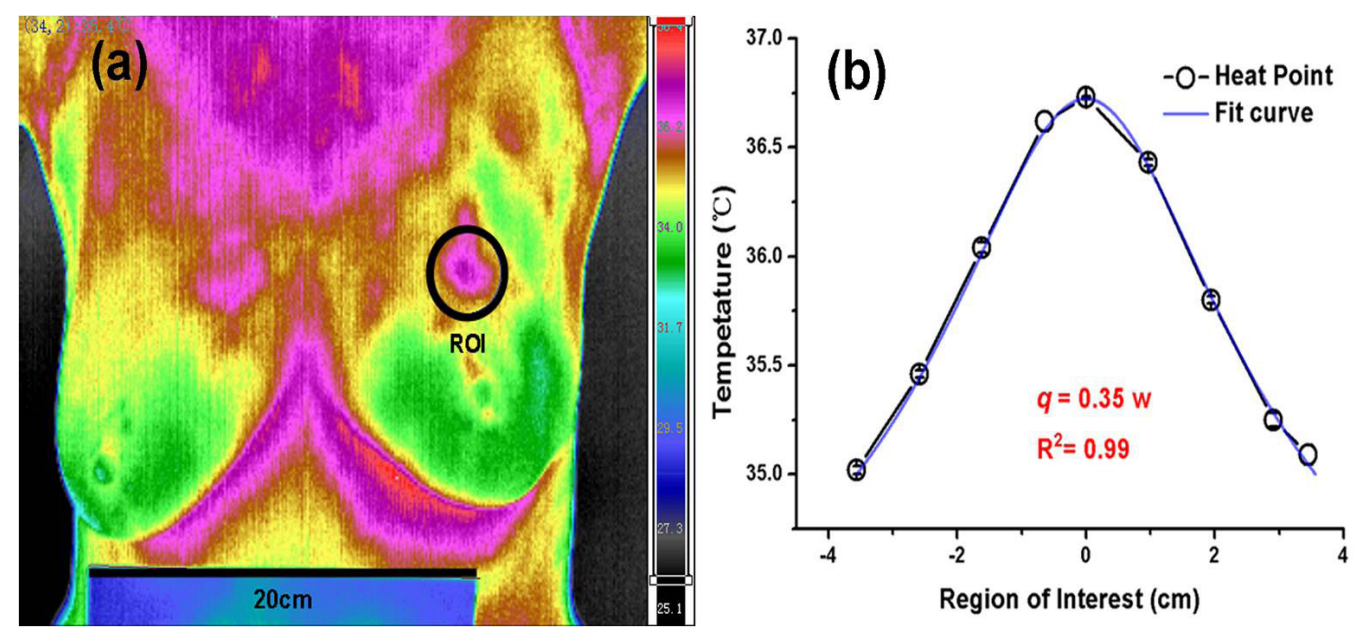

Figure 2. Thermal internal heat source analysis. (a) Infrared imaging and region of interest (ROI); (b) temperature curve and the data fitting results.

Table 2. Results of tests with different $q$-values set as the threshold between breast cancer and benign disease.

\begin{tabular}{|c|c|c|c|c|c|c|}
\hline \multirow[t]{2}{*}{ Test } & \multicolumn{2}{|c|}{$q$-value interval } & \multirow[t]{2}{*}{ Sensitivity (\%) } & \multirow[t]{2}{*}{ False-positive rate $(\%)$} & \multirow[t]{2}{*}{ Specificity (\%) } & \multirow[t]{2}{*}{ Accuracy $(\%)$} \\
\hline & Benign & Cancer & & & & \\
\hline 1 & {$[0.0-0.1]$} & {$[0.1-0.5]$} & 99.6 & 34.9 & 65.1 & 74.6 \\
\hline 2 & {$[0.0-0.2]$} & {$[0.2-0.5]$} & 94.2 & 7.1 & 92.9 & 93.2 \\
\hline 3 & {$[0.0-0.3]$} & {$[0.3-0.5]$} & 76.2 & 2.5 & 97.5 & 91.7 \\
\hline
\end{tabular}

$q$-values of $0.1,0.2$, and 0.3 were set as the threshold values separating breast cancer from benign disease in tests 1,2 , and 3 , respectively.

Table 3. Cross-tabulation of breast cancer identification by internal heat source analysis and breast biopsy.

\begin{tabular}{|c|c|c|c|c|c|}
\hline \multirow[t]{2}{*}{ Breast biopsy } & \multicolumn{3}{|c|}{ Internal heat source analysis } & \multicolumn{2}{|c|}{ Kappa statistics } \\
\hline & Benign & Cancer & Total & $k(95 \% \mathrm{CI})$ & $\mathrm{P}$ \\
\hline Benign & $639(67.4 \%)$ & $49(5.2 \%)$ & $688(72.6 \%)$ & $0.837(0.781-0.873)$ & $<0.001$ \\
\hline Cancer & $15(1.6 \%)$ & $245(25.8 \%)$ & $260(27.4 \%)$ & & \\
\hline Total & $654(69.0 \%)$ & $294(31.0 \%)$ & $948(100 \%)$ & & \\
\hline
\end{tabular}

$95 \% \mathrm{CI}=95 \%$ confidence interval. Cases with analysis results agreeing with biopsy results are shown in bold.

An OR estimate was performed to investigate the influences of breast density and tumor size on the methodological efficiency of the method. In this study, breast density was characterized as either fatty or dense according to the mammography results; most of the patients' characteristics, including age and menopausal status, are displayed in Table 1. Among the 948 women who underwent analysis, 65.1\% (617/948) had fatty breasts and 34.9\% (331/948) had dense breasts (Table 4). Breast density was determined irrelevant to the internal heat source analytical accuracy $(\mathrm{OR}=1.194,95 \% \mathrm{CI}=0.691-2.062, \mathrm{P}=0.524$; Table 4). 
The patients were also divided into 3 groups according to tumor size as follows: T1, $<2 \mathrm{~cm}$; T2, 2 to $5 \mathrm{~cm}$; and T3, $>5 \mathrm{~cm}$ (Table 4). Overall, $87.2 \%(\mathrm{~N}=827 / 948)$ of the women had tumors $<5 \mathrm{~cm}$ in size. The proportions of correct judgments in groups T1, T2, and T3 were 92.5, 93.6, and 91.7\%, respectively. Because the $95 \% \mathrm{CI}$ included 1 , no significant increase in the accuracy rate was observed for group $\mathrm{T} 2(\mathrm{OR}=1.174,95 \% \mathrm{CI}=0.515-2.679)$ relative to $\mathrm{T} 1$, whereas an insignificant inverse association was observed between groups T3 and T1 (OR $=0.893,95 \% \mathrm{CI}=0.327-2.442, \mathrm{P}=0.724)$.

\begin{tabular}{|c|c|c|c|c|c|c|}
\hline \multirow[t]{2}{*}{ Variable } & \multicolumn{2}{|c|}{ All patients } & \multicolumn{2}{|c|}{ Right judgments } & \multirow[t]{2}{*}{ OR $(95 \% \mathrm{CI})$} & \multirow[t]{2}{*}{$P$} \\
\hline & $\mathrm{N}$ & $\%$ & $\mathrm{~N}$ & $\%$ & & \\
\hline Breast density & & & & & & 0.524 \\
\hline Fatty & 617 & 65.1 & 573 & 92.9 & 1.00 (Ref) & \\
\hline Dense & 331 & 34.9 & 311 & 94.0 & $1.194(0.691-2.062)$ & \\
\hline Tumor size & & & & & & 0.724 \\
\hline $\mathrm{T} 1(<2 \mathrm{~cm})$ & 94 & 9.9 & 87 & 92.5 & $1.00(\mathrm{Ref})$ & \\
\hline T2 $(2-5 \mathrm{~cm})$ & 733 & 77.3 & 686 & 93.6 & $1.174(0.515-2.679)$ & \\
\hline $\mathrm{T} 3(>5 \mathrm{~cm})$ & 121 & 12.8 & 111 & 91.7 & $0.893(0.327-2.442)$ & \\
\hline
\end{tabular}

Right judgments denote both patients with benign disease and cancer whose statuses were correctly detected by internal heat source analysis. OR and $\mathrm{P}$ represent odds ratios and $\mathrm{P}$ values, respectively as determined using the Pearson chi-square test. $95 \% \mathrm{CI}=95 \%$ confidence interval.

\section{DISCUSSION}

This study aimed to quantify the relationship between the tumor and skin temperatures. A novel and simple method was developed to simulate the tumor within the breast tissue. Specifically, in this study the tumor was expressed as an equivalent-point heat source, and its thermal power was described as a $q$-value $(0$ to $0.5 w$ ). This method was verified statistically in a clinical trial of 948 patients with breast disease during the period of 2009 to 2013. According to the $q$ value criterion, most of the benign cases fell within the range of 0 to $0.2(639 / 688,92.9 \%)$, and most of the cancer cases lie in the range of 0.2 to $0.5(245 / 260,94.2 \%)$. Note that the findings in this study were similar to those of previous studies in which malignant tumors exhibited higher temperatures than did benign tumors. Subsequently, 3 tests involving the categorical $q$-values of $0.1,0.2$, and 0.3 were performed and the associated error rates (sensitivity, specificity, and accuracy) demonstrated that a $q$-value of 0.2 yielded the best performance so that all of the evaluated rates exceeded $92 \%$. It could therefore be concluded that a thermal power of $0.2 w$ was a fully fit optimal threshold for distinguishing between breast cancer and benign disease.

Pathological confirmation of either breast carcinoma or benign disease via the goldstandard assessment of breast biopsy was a strict requirement for study inclusion. In the statistical analysis, the final outcome was accordingly described as either "cancer" or "benign". Overall, 884 of the 948 assessment results exhibited exact agreement between the internal heat source analysis and breast biopsy results. Furthermore, kappa statistics were assessed for coherence verification so that a $k \geq 0.75$ would indicate a good agreement with the goldstandard assessment and a $k<0.4$ would indicate a poor agreement. The results demonstrated an excellent agreement between the proposed approach and the gold-standard assessment $(k$ $>0.8$ ). Therefore, an internal $q$-value in the range of 0.3 to 0.5 could be considered as an area 
at risk of breast cancer. Among all of the "cancer" cases $(\mathrm{N}=294)$ judged using the approach proposed in this study, 245 were true cancer cases (confirmed by biopsy); the determinations of 49 cases failed. Notably, the sensitivity was far greater than the false-positive rate $(94.2 v s$ $7.1 \%)$. This result provided further evidence of an association between a higher $q$-value ( $q>$ $0.2 w)$ and the breast cancer risk.

In consideration of the effects of breast density and tumor size on accuracy of this approach, statistical analyses involving these factors were also discussed in our study. Earlier studies demonstrated that breast density could be described as a function of a woman's age that reduces as the woman's age increases (Kerlikowske et al., 1996; Niklason et al., 1998). In general, breast density is categorized as 1) entirely fat, 2) scattered fibroglandular densities, 3) heterogeneously dense, or 4) extremely dense (Razzaghi et al., 2012). Normally, this factor is dichotomized as either fatty (categories 1 and 2) or dense (categories 3 and 4) when conducting large-scale statistical analyses (Kim et al., 2013). In this study, of the 948 women who underwent analysis, 617 and 331 cases were classified as having fatty and dense breast tissue, respectively. The chi-square test results suggested the lack of a significant difference between these groups $(\mathrm{P}>0.5)$. Furthermore, the results of the $\mathrm{OR}$ estimates suggested that the accuracy of breast cancer detection via the method implemented in this study did not correlate significantly with the presence of dense breasts versus fatty breasts $(\mathrm{OR} \approx 1)$.

Cancers diagnosed at advanced stages have sufficiently large volumes to render the diseases less treatable. A tumor size-independent detection method would provide more advantages than would common imaging methods such as mammography. Therefore, the impact of tumor size on our method should be considered. In each size group (T1, $<2 \mathrm{~cm} ; \mathrm{T} 2,2$ to 5 $\mathrm{cm} ; \mathrm{T} 3,>5 \mathrm{~cm}$ ), agreement between the biopsy and proposed method was confirmed for $>90 \%$ of the analytical results. Moreover, because CI included 1, the results of the OR estimates suggest that the proposed method is independent of tumor size.

Finally, a comparative thermal analysis of internal heat source using a common infrared diagnostic technique was discussed. Common infrared diagnostic techniques merely indicate hot spots (i.e., higher temperatures) on the skin that indicate the presence of an abnormality in deeper tissues. These diagnoses are based on only 2D infrared images, and the fuzzy tumor tissue images are acquired. In comparison, the internal heat source parameter (e.g., thermal power $q$ ) could be determined via an internal heat source analysis. Cancer or benign disease could be identified from the corresponding $q$-value. Subsequently, a quantitative analysis of the tumor characteristics could be realized from the internal heat source analysis, which serves as a functional method for breast cancer detection.

In summary, we have quantified the relationship between the cancer diagnosis and infrared images using an internal heat source analysis, which was scarcely obtainable through previous studies. These results reflect the heat generated by the increased metabolism of the embedded tumor and provide a functional imaging technique that will considerably facilitate breast cancer diagnosis. The present study suggests that an internal heat source-based analysis is independent of the breast density and tumor size. This technique could be considered a safe, feasible, and effective diagnostic solution for detecting cancerous tumors with a broad clinical application scope.

\section{Conflict of interest}

The authors declare no conflicts of interests. 


\section{ACKNOWLEDGMENTS}

Research supported by grants from the National Major Special Program of Scientific Instrument and Equipment Development of China (\#2012YQ160203); the first author is also grateful for financial support from the Fundamental Research Funds for the Center Universities of China (\#2011120202020006).

\section{REFERENCES}

AbdullGaffar B, Ghazi E, Mohamed E and Hamza D (2012). Breast metaplastic carcinoma with unusual small cell component. Breast Dis. 34: 19-24.

Absalan H, SalmanOgli A, Rostami R and Maghoul A (2012). Simulation and investigation of quantum dot effects as internal heat-generator source in breast tumor site. J. Therm. Biol. 37: 490-495.

Aksel EM and Mikhailov EA (2005). Morbidity statistics of breast cancer in Moscow. Voprosy onkologii 51: 656-658.

Allan AL, Vantyghem SA, Tuck AB and Chambers AF (2006). Tumor dormancy and cancer stem cells: implications for the biology and treatment of breast cancer metastasis. Breast Dis. 26: 87-98.

Alroy I and Yarden Y (2000). Biochemistry of HER2 oncogenesis in breast cancer. Breast Dis. 11: 31-48.

Anbar M (1998). Clinical thermal imaging today. IEEE Eng. Med. Biol. Mag. 17: 25-33.

Anderson WF, Schairer C, Chen BE, Hance KW, et al. (2005). Epidemiology of inflammatory breast cancer (IBC). Breast Disease 22: 9-23.

Aupperlee M, Kariagina A, Osuch J and Haslam SZ (2005). Progestins and breast cancer. Breast Dis. 24: 37-57.

Bezić J, Mrklić I, Pogorelić Z and Tomić S (2013). Mammographic screening has failed to improve pathohistological characteristics of breast cancers in Split region of Croatia. Breast Dis. 34: 47-51.

Brown KL, Moglia DM and Grumet S (2006). Genetic counseling for breast cancer risk: general concepts, challenging themes and future directions. Breast Dis. 27: 69-96.

Carter P, Fendly BM, Lewis GD and Sliwkowski MX (2000). Development of herceptin. Breast Dis. 11: 103-111.

Chandrasekar SGC, Rathi P and Aggarwal R (2010). Organophosphate poisoning presenting as acute respiratory distress. Indian J. Pediatr. 77: 1195-1196.

Chavez KJ, Garimella SV and Lipkowitz S (2010). Triple negative breast cancer cell lines: one tool in the search for better treatment of triple negative breast cancer. Breast Dis. 32: 35-48.

Choudhury A and Kiessling R (2004). Her-2/neu as a paradigm of a tumor-specific target for therapy. Breast Dis. 20: 25-31.

Chunfang G, Kaiyang L and Shaoping Z (2005). A Novel Approach of Analyzing the Relation Between the Inner Heat Source and the Surface Temperature Distribution in Thermal Texture Maps. Conference Proceedings. Annual International Conference of the IEEE Engineering in Medicine and Biology Society. IEEE Engineering in Medicine and Biology Society, 1: 623-626.

Draper JW and Jones CH (1969). Thermal patterns of the female breast. Br. J. Radiol. 42: 401-410.

Erb KM, Shapiro-Wright HM and Julian TB (2010). Axillary recurrences following positive sentinel lymph node biopsy with individual tumor cells or micrometastases and no axillary dissection. Breast Dis. 31: 83-90.

Farese SA and Aebi S (2008). Infiltrating lobular carcinoma of the breast: systemic treatment. Breast Dis. 30: 45-52.

Feasey CM, James WB and Davison M (1970). A technique for breast thermography. Br. J. Radiol. 43: 462-465.

Gautherie M (1980). Thermopathology of breast cancer: measurement and analysis of in vivo temperature and blood flow. Ann. N. Y. Acad. Sci. 335: 383-415.

Gescheit IM, Dayan A, Ben-David M and Gannot I (2010). Minimal-invasive thermal imaging of a malignant tumor: a simple model and algorithm. Med. Phys. 37: 211-216.

He Y, Zheng X, Sit C, Loo WT, et al. (2012). Using association rules mining to explore pattern of Chinese medicinal formulae (prescription) in treating and preventing breast cancer recurrence and metastasis. J. Transl. Med. 10: S12.

Jemal A, Murray T, Ward E, Samuels A, et al. (2005). Cancer statistics, 2005. CA Cancer J. Clin. 55: 10-30.

Jones BF and Plassmann P (2002). Digital infrared thermal imaging of human skin. IEEE Eng. Med. Biol. Mag. 21: 41-48.

Kerlikowske K, Grady D, Barclay J, et al. (1996). Effect of age, breast density, and family history on the sensitivity of first screening mammography. JAMA 276: 33-38.

Kim WH, Moon WK, Kim SJ, Yi A, et al. (2013). Ultrasonographic assessment of breast density. Breast Cancer Res. Treat. 138: 851-859.

Lawson RN and Gaston JP (1964). Temperature measurements of localized pathological processes. Ann. N. Y. Acad. Sci. 121: $90-98$. 
Lawson RN and Alt LL (1965). Skin temperature recording with phosphors: a new technique. Can. Med. Assoc. J. 92: 255-260.

Liu KC and Lin CN (2010). Temperature prediction for tumor hyperthermia with the behavior of thermal wave. Numer. Heat Transf. Appl. 58: 819-833.

Niklason LT, Kopans DB and Hamberg LM (1998). Digital breast imaging: tomosynthesis and digital subtraction mammography. Breast Dis. 10: 151-164.

Pennes HH (1948). Analysis of tissue and arterial blood temperatures in the resting human forearm. J. Appl. Physiol. 1: 93-122.

Qian X, Tucker A, Gidcumb E, Lu J, et al. (2012). A stationary digital breast tomosynthesis scanner. Conference paper. SPIE Proceedings 8313.

Razzaghi H, Troester MA, Gierach GL, Olshan AF, et al. (2012). Mammographic density and breast cancer risk in White and African American Women. Breast Cancer Res. Treat. 135: 571-580.

SalmanOgli A and Rostami A (2012). Modeling and improvement of breast cancer site temperature profile by implantation of onion-like quantum-dot quantum-well heteronanocrystal in tumor site. IEEE Trans. Nanotech. 11: 1183-1191.

Siegel R, Naishadham D and Jemal A (2013). Cancer statistics, 2013. CA Cancer J. Clin. 63: 11-30.

Ye F and Shi GL (2013). Clinical breast cancer analysis with surface fitting in the medical thermal texture maps. Appl. Mech. Mater. 263: 2454-2457.

Zastrow E, Hagness SC and Van Veen BD (2010). 3D computational study of non-invasive patient-specific microwave hyperthermia treatment of breast cancer. Phys. Med. Biol. 55: 3611-3629. 